\title{
Endograft connector technique to treat popliteal artery aneurysm in a morbid obese patient
}

\author{
Felice Pecoraro, Guido Bajardi, Ettore Dinoto, Gaetano Vitale, \\ Mario Bellisi and Umberto Marcello Bracale
}

\begin{abstract}
Surgical repair of popliteal artery aneurysm in morbid obese patients poses additional challenges. We report a morbid obese patient who had a $59 \mathrm{~mm}$ right popliteal artery aneurysm which was successfully treated with the endograft connector technique. This technique was used to perform the distal anastomosis of the below-knee femoro-popliteal bypass. A $10 \mathrm{~mm}$ Dacron graft was used as a main graft bypass and an $11 \mathrm{~mm} / 10 \mathrm{~cm}$ stentgraft as endograft connector. Following the respective tunnel of the Dacron graft, an end-to-side proximal anastomosis was performed at distal femoral artery. The aneurysm exclusion was obtained through a proximal and a distal ligation. Postoperative duplex showed adequate bypass patency. Knee x-rays demonstrated no signs of stent kinking/fractures. The postoperative course was uneventful and the patient was discharged home on fourth day post operative. The six-month computed tomography scan and the 12-month duplex control showed a patent bypass with no signs of stenosis.
\end{abstract}

\section{Keyword}

Popliteal artery aneurysm, femoro-popliteal bypass, sutureless anastomosis

\section{Introduction}

The concept of endograft connectors (ECs) was first introduced by Carrel in 1984 to create vascular anatomoses. ${ }^{1}$ Recently, ECs have been increasingly used and reported in the medical literature, especially for revascularization of the visceral arteries. ${ }^{2-6}$ However, only the recent improvements in techniques and medical devices have allowed the use of such anastomotic concept in vascular surgery. Herein, we report a case when an EC was employed to perform the distal anastomosis in a below-knee (BK) femoro-popliteal (fem-pop) bypass in a morbid obese patient who had a large symptomatic popliteal artery aneurysm (PAA).

\section{Technique}

A 63-year-old man with type II diabetes was referred to our Vascular Surgery Unit due to acute pain in the right leg. The patient had reported similar symptoms six months before, which resolved spontaneously. The physical examination revealed that the patient was morbid and obese with a body mass index of 48.7, bilateral palpable femoral and distal pulses in the lower extremities and a pulsatile mass in the right popliteal fossa. Duplex examination showed a $59 \mathrm{~mm}$ PAA and a computed tomography angiography (CTA) confirmed the findings (Figure 1(a)). A BK fem-pop bypass was deemed necessary due to the risk of PAA rupture and distal embolization, and a signed informed consent was obtained prior the procedure.

A $10 \mathrm{~mm}$ Dacron graft was used as main bypass graft due to the lack of suitable great saphenous veins (GSV) as per duplex examination. Both bypass anastomoses zones were preoperatively identified on CT. The proximal anastomosis was planned at the level of distal superficial femoral artery. A suitable distal landing zone was identified in correspondence of BK popliteal artery distally to the PAA. At this level, the distance from skin to target artery was $94 \mathrm{~mm}$ (Figure 1(b)). First, a surgical isolation of both proximal and distal

Vascular Surgery Unit, University Hospital 'P. Giaccone', Palermo, Italy

\section{Corresponding author:}

Felice Pecoraro, Vascular Surgery Unit, University Hospital “P. Giaccone”, Via Liborio Giuffrè 5, 90127 Palermo, Italy.

Email: felicepecoraro@libero.it 


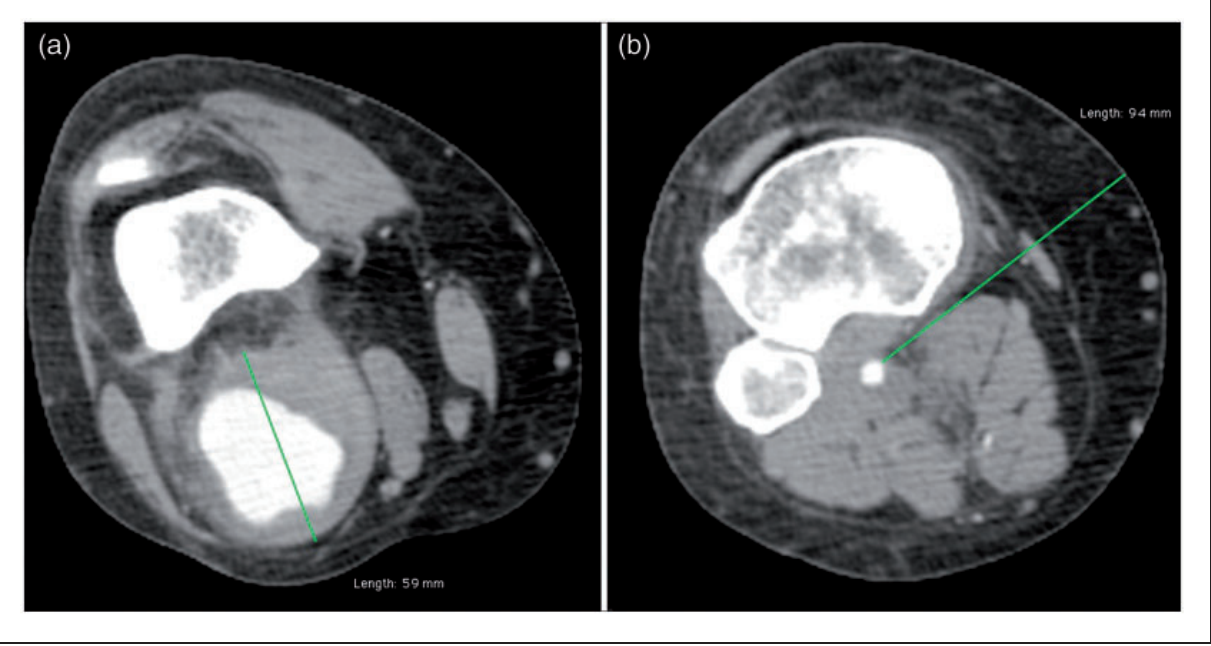

Figure I. Preoperative computed tomography angiography. (a) Popliteal artery aneurysm maximal transverse diameter. (b) Distal anastomosis target artery: depth from skin of below-knee popliteal artery.

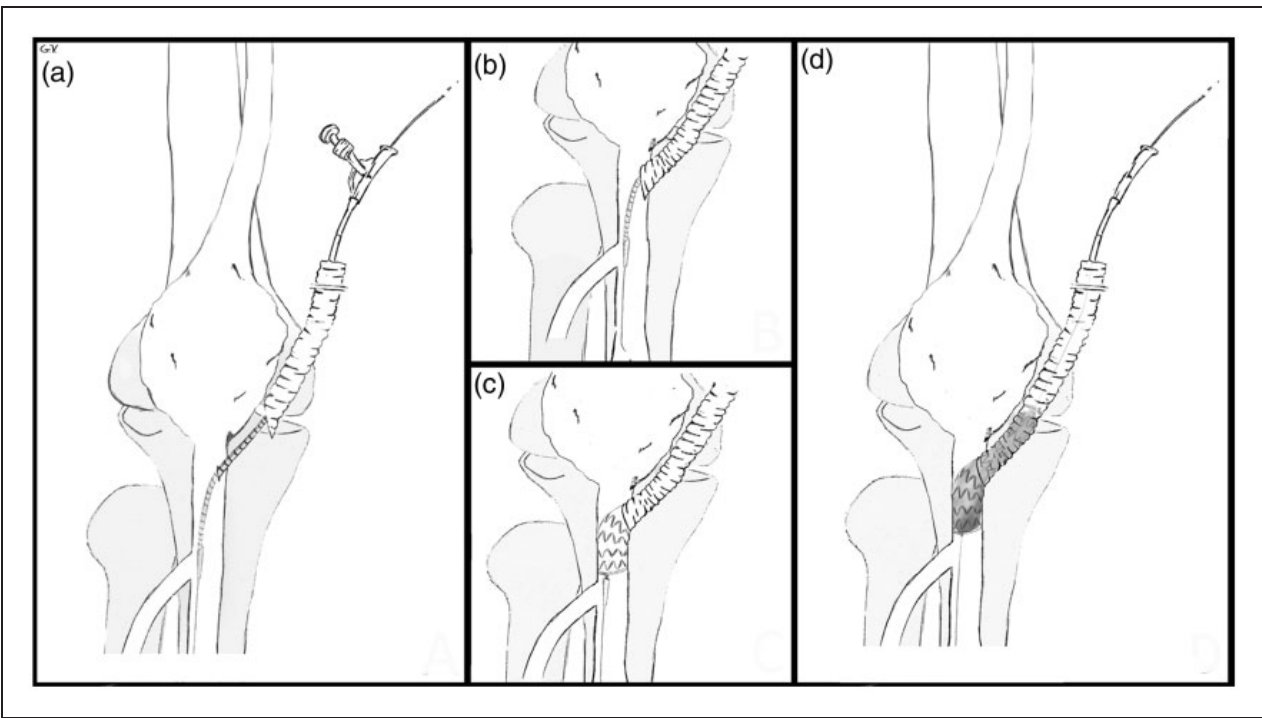

Figure 2. Intraoperative view. (a) Endograft connector introduced into popliteal artery. (b) Main graft positioning, over the endograft connector, in contact with popliteal artery.

bypass anastomotic zone was accomplished. The EC connector has been previously reported to be applied to supraaortic, visceral and above-knee arteries. ${ }^{2-7}$ For BK popliteal artery, an analogous technique was employed for distal anastomosis. An $11 \mathrm{~mm} / 10 \mathrm{~cm}$ ViaBahn (W.L. Gore, Flagstaff, USA) stentgraft was used as EC between the Dacron graft and the BK popliteal artery (Figure 2). Briefly, the EC is marked with a sterile pen at $2 \mathrm{~cm}$ from the distal end and introduced coaxially into the main graft. The isolated distal popliteal artery is then punctured with a needle and a $0.035 \mathrm{in}$. guide wire is advanced into it. After removing the needle, the distal side of the wire is introduced in a retrograde fashion into the EC (previously introduced into the main graft). The $\mathrm{EC}$ is advanced, over the wire, for $2 \mathrm{~cm}$ into the popliteal artery (Figure 3(a)). Then, the main graft is moved over the EC to reach its position in contact with the popliteal artery (Figure 3(b)). Once in position, the EC is deployed and a balloon is inflated at the anastomosis level over the same guide wire. The intrinsic radial force of the EC (self-expandable stent graft) in association with the EC ballooning are responsible for the EC sealing at the level of both the main graft and the target artery (Figure 3(c)). In this case, at balloon deflation, the run-off from popliteal artery was consistent and no bleeding was observed 


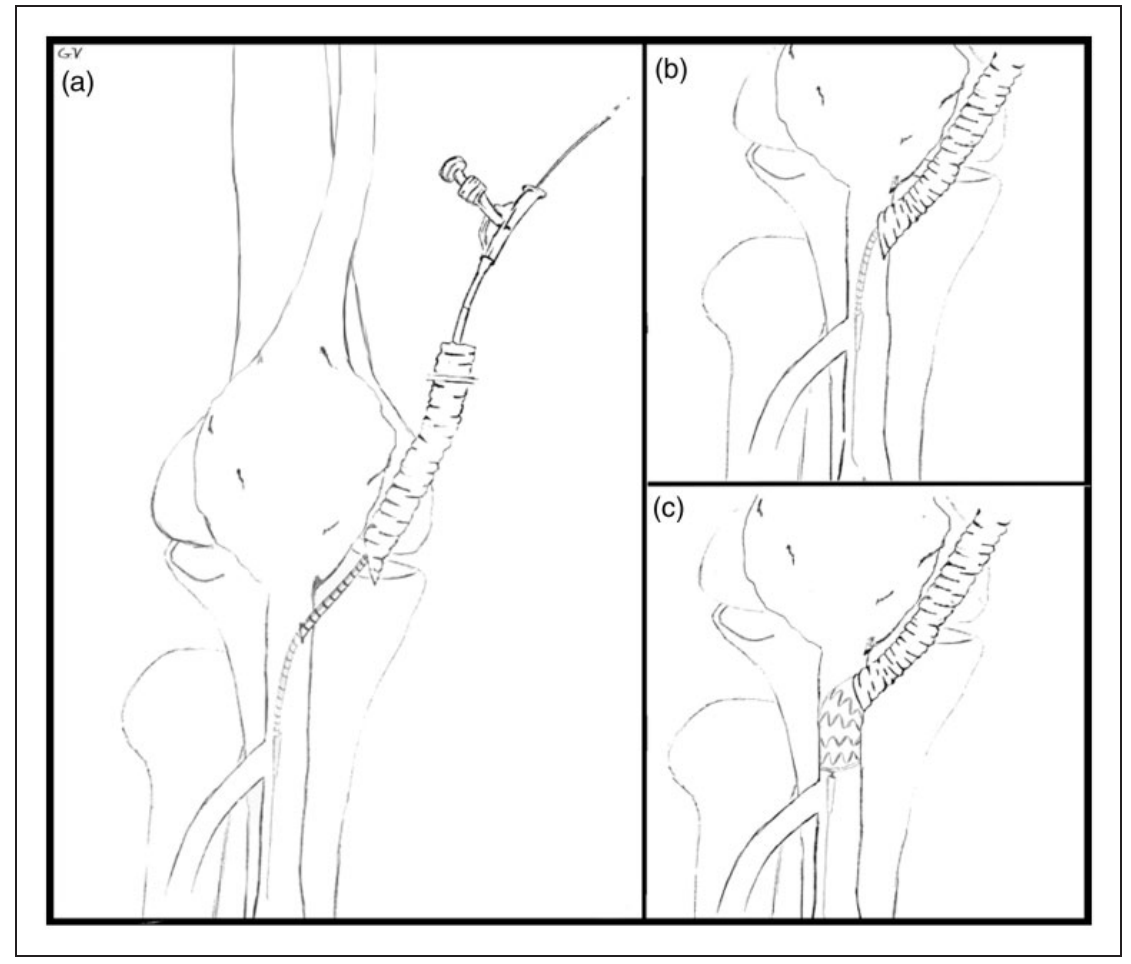

Figure 3. Endograft connector technique. (a) Endograft connector through main graft introduced into popliteal artery. (b) Main graft positioning, over the endograft connector, in contact with popliteal artery. (c) Endograft connector deployment. (d) Endograft connector ballooning and full expansion with complete sealing in correspondence of the popliteal artery and main graft.

from the EC. A single Prolene stitch was placed to fix the EC, the main graft and the popliteal artery at the same time. The time required to perform the distal anastomosis was less than $5 \mathrm{~min}$. To accomplish the bypass procedure the main graft was tunnelled through the popliteal fossa, the length was corrected and an end-to-side anastomosis to the distal femoral artery was performed. The PAA exclusion was obtained through a distal ligation to proximal anastomosis and a proximal ligation to distal anastomosis. Postoperative duplex showed adequate bypass patency and preserved distal flow in all distal arteries. Antero-posterior and lateral $\mathrm{x}$-ray of the knee were taken in extension (including both antero-posterior and lateral views) and in $90^{\circ}$ flexion. These $\mathrm{x}$-rays showed no signs of stentgraft kinking or fracture (Figure 4). The postoperative course was uneventful and the patient was discharged home on day 4 postoperative. The sixmonth CT scan revealed a patent bypass with no signs of stenosis or stentgraft fracture (Figure 5). Patency is maintained at 12-month duplex control.

\section{Comment}

PAAs repair is recommended for all symptomatic patients. For asymptomatic PAAs, a maximum diameter $>2 \mathrm{~cm}$ is considered as an indication for treatment. ${ }^{8}$

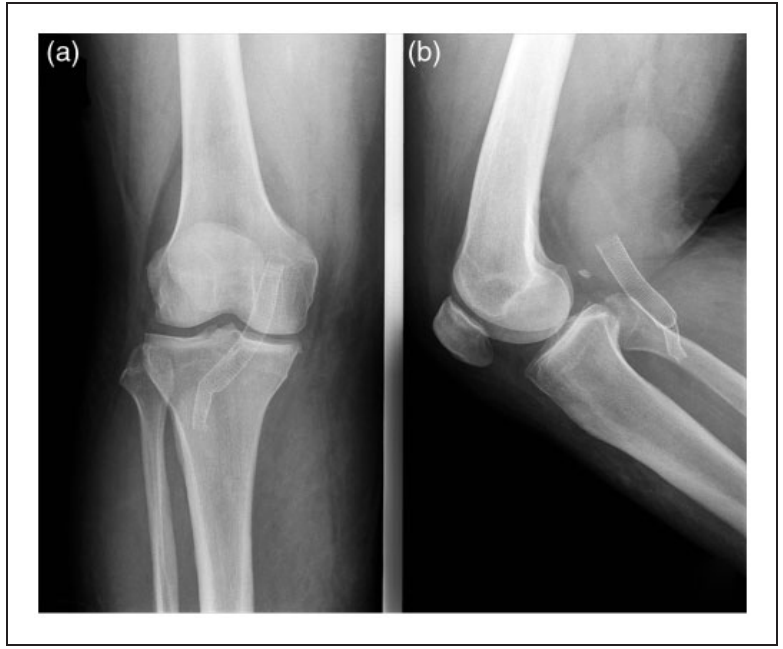

Figure 4. Radiographs of the knee. (a) Antero-posterior view. (b) Lateral view with $90^{\circ}$ joint flexion.

PAAs are usually treated with GSV fem-pop bypass through a medial approach ${ }^{9}$; or, in case of PAAs limited extension, by posterior approach. ${ }^{8-10}$ Endovascular treatment has been recently introduced as a consistent alternative to surgical therapy with acceptable results. Tielliu et al. ${ }^{11}$ reported the first results with endovascular repair of PAAs and showed slightly inferior 


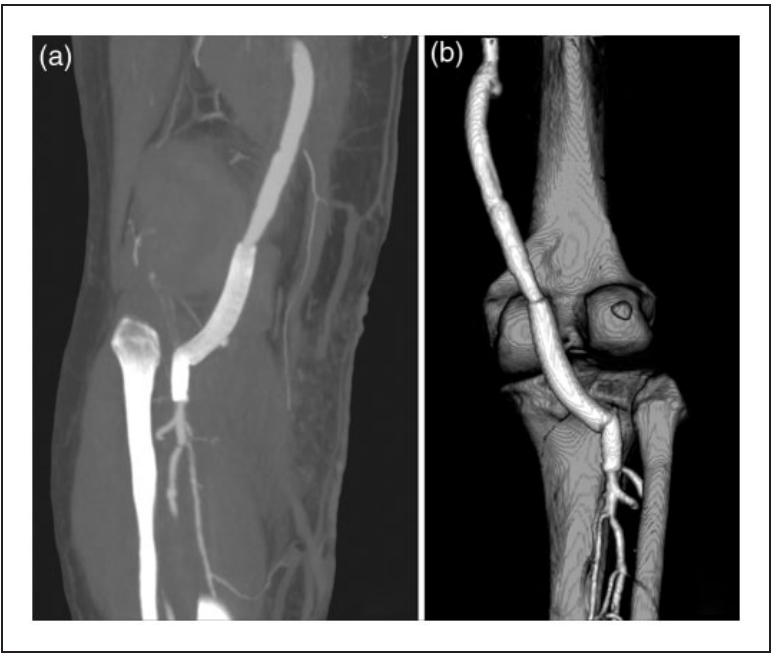

Figure 5. Six-month computed tomography angiography. (a) MPR showing complete aneurysm exclusion. (b) VR posterior view showing endograft connector and tibial arteries patency.

outcomes compared to open surgery, but with reduced invasiveness and shortened hospital stay. Larger series with long-term results of this approach need to be validated. In the present report, the preoperative balance favours for an open treatment due to the relatively young age of the patient. Moreover, an endovascular attempt was excluded due to the risk of passing wires through a PAA embolic thrombus. In this case, the major concern of surgical treatment was related to the depth of the distal anastomotic zone in correspondence to the distal popliteal artery due to the patient's morbid obese condition. The need of a large dissection to obtain an acceptable surgical exposure and perform the distal anastomosis, was preoperatively evident. Maintaining the same rationale of the BK fem-pop bypass for PAA treatment, the distal anastomosis was performed with the EC technique to avoid an extensive surgical preparation and reduced anastomosis difficulty. The concept of traditional suture anastomosis has been historically considered the gold standard to create a vascular anastomosis. The EC technique has been recently employed to reduce bleeding and clamping time in selected cases, when less ischemia or dissection is mandatory. Its use has been reported also when to clamp or to perform a traditional suture anastomosis is difficult or impossible. ${ }^{12,13}$ Although the goal of this report is not to compare the EC technique to the traditional anastomosis, the EC technique seems to be effective and consistently reduced the challenge to perform the distal anastomosis. Another minor issue of the EC technique is related to the absence of suture hole bleeding related to traditional anastomosis, especially with PTFE (Polytetrafluoroethylene) use. ${ }^{14}$ In the preoperative CTA, the popliteal artery was seen $94 \mathrm{~cm}$ distal from the skin. This finding was intraoperatively confirmed and a high discomfort to perform a traditional distal anastomosis was evident. Adopting the EC technique, the exposition of a short BK popliteal artery portion was enough to perform distal anastomosis. Moreover, a reduction in operative and anaesthesia time was obtained. The interest in finding alternative ways to achieve vascular anastomosis was catalyzed by the introduction, in 2011, of the GORE Hybrid Vascular Graft (W. L. Gore \& Associates, Flagstaff, AZ). ${ }^{15}$ Using the same rationale of EC technique, the hybrid graft could allow an easier and faster approach to such anastomosis. Large series and long-term results are mandatory to validate the EC technique.

\section{Conclusion}

The EC technique is a feasible approach in BK anastomosis. The limited experience with this technique allows its use in specific situations such as difficult or prohibited dissection or anastomosis. A relevant limitation to a widespread use of this technique is related to EC costs. Mid- and long-term results are expected to validate the EC technique.

\section{Conflict of interest}

None declared.

\section{Funding}

This research received no specific grant from any funding agency in the public, commercial, or not-for-profit sectors.

\section{References}

1. Zeebregts CJ, Heijmen RH, van den Dungen JJ, et al. Non-suture methods of vascular anastomosis. $\mathrm{Br} J$ Surg 2003; 90: 261-271.

2. Lachat M, Mayer D, Criado FJ, et al. New technique to facilitate renal revascularization with use of telescoping self-expanding stent grafts: VORTEC. Vascular 2008; 16 : 69-72.

3. Rancic Z, Mayer D, Pfammatter T, et al. A new sutureless telescoping anastomotic technique for major aortic branch revascularization with minimal dissection and ischemia. Ann Surg 2010; 252: 884-889.

4. Donas KP, Rancic Z, Lachat M, et al. Novel sutureless telescoping anastomosis revascularization technique of supra-aortic vessels to simplify combined open endovascular procedures in the treatment of aortic arch pathologies. J Vasc Surg 2010; 51: 836-841.

5. Donas KP, Lachat M, Rancic Z, et al. Early and midterm outcome of a novel technique to simplify the hybrid procedures in the treatment of thoracoabdominal and pararenal aortic aneurysms. J Vasc Surg 2009; 50: 1280-11284.

6. Greenberg G, Szendro G, Mayzler O, et al. Use of ViaBahn open revascularisation technique for aboveknee femoro-popliteal anastomosis: a technical note. Eur J Vasc Endovasc Surg 2011; 42: 202-205. 
7. Szendro G, Greenberg G, Leytzin A, et al. A new minimally invasive hybrid technique for femoro - above knee popliteal bypass. Int Angiol 2011; 30: 522-526.

8. Bracale UM, Corte G, Di Gregorio A, et al. Surgical repair of popliteal artery aneurysms remains a safe treatment option in the endovascular era: a 10-year singlecenter study. Ann Ital Chir 2011; 82: 443-448.

9. Bellosta R, Sarcina A, Luzzani L, et al. Fate of popliteal artery aneurysms after exclusion and bypass. Ann Vasc Surg 2010; 24: 885-889.

10. Bajardi G, Pecoraro F, Vitale G, et al. A popliteal artery aneurysm presenting with ab extrinseco popliteal vein occlusion and compartment syndrome: a case report. Ann Ital Chir 2012; 83: 441-444.

11. Tielliu IF, Verhoeven EL, Zeebregts CJ, et al. Endovascular treatment of popliteal artery aneurysms: results of a prospective cohort study. J Vasc Surg 2005; 41: $561-567$.
12. Lachat M, Mayer D, Pecoraro F, et al. Video presentation sutureless clampless telescoping method for aortic and arterial anastomoses using an endograft connector. J Vasc Surg 2011; 53: 29S.

13. Papadimitriou D, Mayer D, Lachat M, et al. A clampless and sutureless aortic anastomosis technique using an endograft connector for aortoiliac occlusive disease in which the aorta cannot be clamped or sewn due to calcification or scarring. Vascular 2012; 20: 262-267.

14. Bajardi G, Pecoraro F and Mirabella D. Efficacy of TachoSil patches in controlling Dacron suture-hole bleeding after abdominal aortic aneurysm open repair. J Cardiothorac Surg 2009; 4: 60.

15. WL Gore \& Associates, Flagstaff, AZ. http://www. goremedical.com. 2011 (accessed 2 January 2013). 